\title{
SEISMOLOGY OF SUN AND STARS AND RELATED OPACITY LABORATORY MEASUREMENTS
}

\author{
S. Turck-Chièze ${ }^{1}$
}

\begin{abstract}
CNES, ESA and NASA have invested in helioseismic and asteroseismic disciplines for 2 decades with SoHO (1995-2015), COROT (2006-2013), KEPLER (2009-2014), PICARD (2010-2013) and SDO (2010-2015). These missions focus on the stellar internal dynamics and their influence of neighboring planets. Progress along this path requires that the microscopic physics is well under control, but several seismic probes indicate some discrepancies which justify new investigations of the energy transport in radiative zones of the Sun and massive stars, despite strong efforts dedicated to reaction rates, screening, equation of state and opacity coefficients between 1990 and 2000. We describe here how the OPAC consortium tackles the complex problem of photon absorption by matter both theoretically and experimentally, by using high energy laser facilities. These studies might be also useful for other disciplines like fusion for energy and X-ray spectroscopy astronomy.
\end{abstract}

\section{Introduction: The seismic approach to improve stellar evolution}

Asteroseismic investigation of stars strongly developed since the European launch of COROT in 2006 and the American satellite KEPLER (2009-2014) succeeding the golden age of helioseismology with SoHO (1995-2015). We observe now several thousands of stars and detect millions of modes for a large range of mass (at least 1 to $15 M_{\odot}$ and even higher). Acoustic and gravity mode frequencies are extracted from the Fourier transform of the radial velocity variability, measured in solar specific lines (SoHO/GOLF and SoHO/MDI: Gabriel et al. 1995; Scherrer et al. 1995, and SDO/HMI: Scherrer et al. 2012), the same technique is used on ground in asteroseismology by adding several lines (Bedding et al. 2001, 2010; Martic et al. 2004) for individual stars. But the most popular technique, used both in COROT (Baglin et al. 2006) and KEPLER (Gilliland et al. 2010), is to extract the Fourier transform spectrum from the variability of the stellar photometry (Chaplin et al. 2010), as thousands of stars are observed simultaneously.

${ }^{1}$ SAp/IRFU/DSM CE Saclay, 91191 Gif-sur-Yvette Cedex, France

(C) The Author(s) 2013

DOI: $10.1051 /$ eas/1258006 

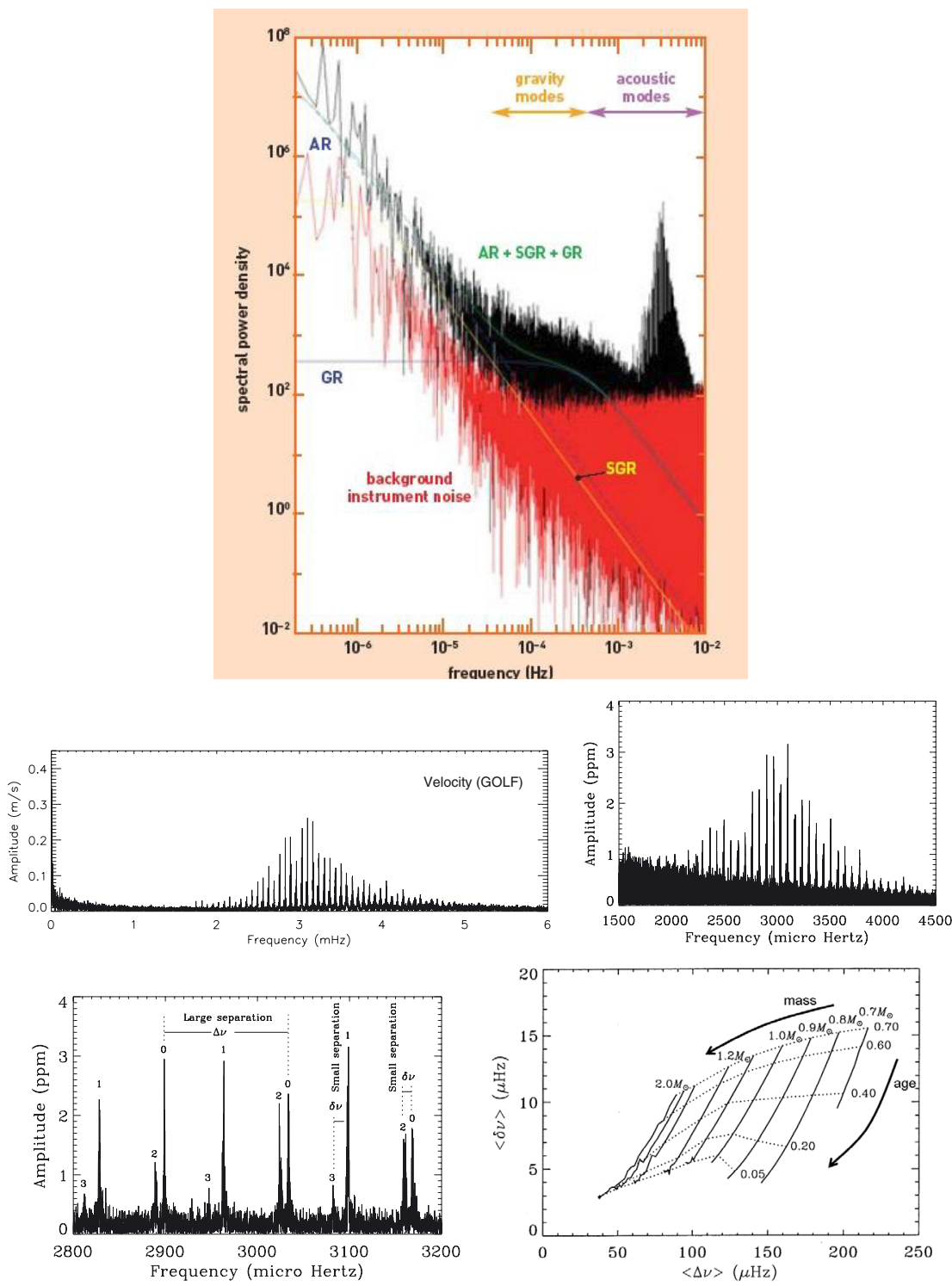

Fig. 1. a) Fourier transform power spectrum of the solar GOLF residual velocity time series, showing the respective ranges of gravity and acoustic modes together with the contribution of the solar noise due to the activity of the Sun (granulation + active regions). From Turck-Chièze et al. (2004). b) and c) Zoom on the acoustic modes for velocity (GOLF/SoHO) and intensity (VIRGO/SoHO) measurements. d) Characteristics of the solar-like spectra: mean small versus large differences or separations. e) Seismic diagram for stars. From respectively Christensen-Dalsgaard (2003) and Stello et al. (2007). See text for explanations. 
The Sun is a unique star, observed both locally and globally, so millions of modes are detected which penetrate more or less deep from the surface into the core. The other stars are only observed globally, so only the radial, dipolar and quadrupolar stellar modes are observed (except for deformed stars). The mode frequencies $\nu_{n, \ell, m}$ are labeled by their order $\mathrm{n}$ (number of nodes along the radius) and their degree $\ell$ (number of reflexions at the surface). Theoretical predictions are necessary to identify them. In presence of internal rotation and magnetic field, the $\ell$ component is split in $2 \ell+1$ azimuthal orders $m$. Figure 1 shows (a) the solar power spectrum obtained with the GOLF instrument. The comparison of the velocity (b: GOLF) and intensity (c: VIRGO) spectra from SoHO shows the superiority of the first technique, the surface turbulence induces a noise in the low frequency part of the intensity spectrum. Figure 1d shows the characteristics of the acoustic spectrum for Sun and solar-like stars: the orders of the same degree present a nearly regular spaced comb pattern in frequency following an asymptotic behavior, called the large difference $\Delta \nu$, the phase shift $\alpha$ is largely dependent on the physics of the subsurface and of the EOS of helium and hydrogen:

$$
\nu_{n, \ell}=\left(n+\frac{\ell}{2}+\frac{1}{4}+\alpha\right) \Delta \nu \text { where } \Delta \nu=\left[2 \int_{0}^{\mathrm{R}} \frac{\mathrm{dr}}{\mathrm{c}}\right]^{-1} .
$$

The relation between $\Delta \nu$ and the sound speed $\mathrm{c}$ is extremely useful to label the frequencies. A second quantity, the small separation, i.e. the distance between successive orders and degrees differing by 2 , is also useful for solar-like stars:

$$
\delta \nu_{n, \ell}=\nu_{n, \ell}-\nu_{n-1, \ell+2} \approx-(4 \ell+6) \frac{\Delta \nu}{4 \pi^{2} \nu_{n, \ell}} \int_{0}^{R} \frac{d c}{r} \frac{d r}{r} .
$$

This quantity is sensitive to the core of the star, while the large difference is mainly sensitive to the layers below the surface. The two quantities $\Delta \nu$ and $\delta \nu$ allow to place the star in the seismic diagram from which one deduces an estimate of its mass and age (Fig. 1e). Figure 2a shows the small separation between radial and quadrupolar acoustic modes for 1700 solar-like stars observed by Kepler from the main sequence to the red clump (Huber et al. 2011). The evolution of stars is clearly visible. Moreover, in using the well established laws (Kjeldsen et al. 2008):

$$
\begin{gathered}
\frac{\Delta \nu}{\Delta \nu_{\odot}} \approx\left(\frac{M}{M_{\odot}}\right)^{0.5}\left(\frac{R}{R_{\odot}}\right)^{-1.5} \frac{\nu_{\max }}{\nu_{\max , \odot}} \approx \frac{M}{M_{\odot}}\left(\frac{T_{\text {eff }}}{T_{\text {eff }, \odot}}\right)^{-0.5}\left(\frac{R}{R_{\odot}}\right)^{-2} \\
\frac{A_{\max }}{A_{\max , \odot}} \approx \frac{L / L_{\odot}}{M / M_{\odot}}\left(\frac{T_{\text {eff }}}{T_{\text {eff }, \odot}}\right)^{-0.5}
\end{gathered}
$$

and large differences obtained with an accuracy of $0.5 \%$, small separation at $10 \%$ and $T_{\text {eff }}$ at $2 \%$, one determines radius at $3 \%, \mathrm{M}$ at $5 \%$ and age at about $10 \%$, which represent a real progress (Stello et al. 2007) assuming that the microscopic physics is correct. Here $\nu_{\max }$ represents the maximum of the acoustic peak of the power spectrum, about $5 \mathrm{mn}$ or $3000 \mu \mathrm{Hz}$ for the Sun, see Figure 1a. The 

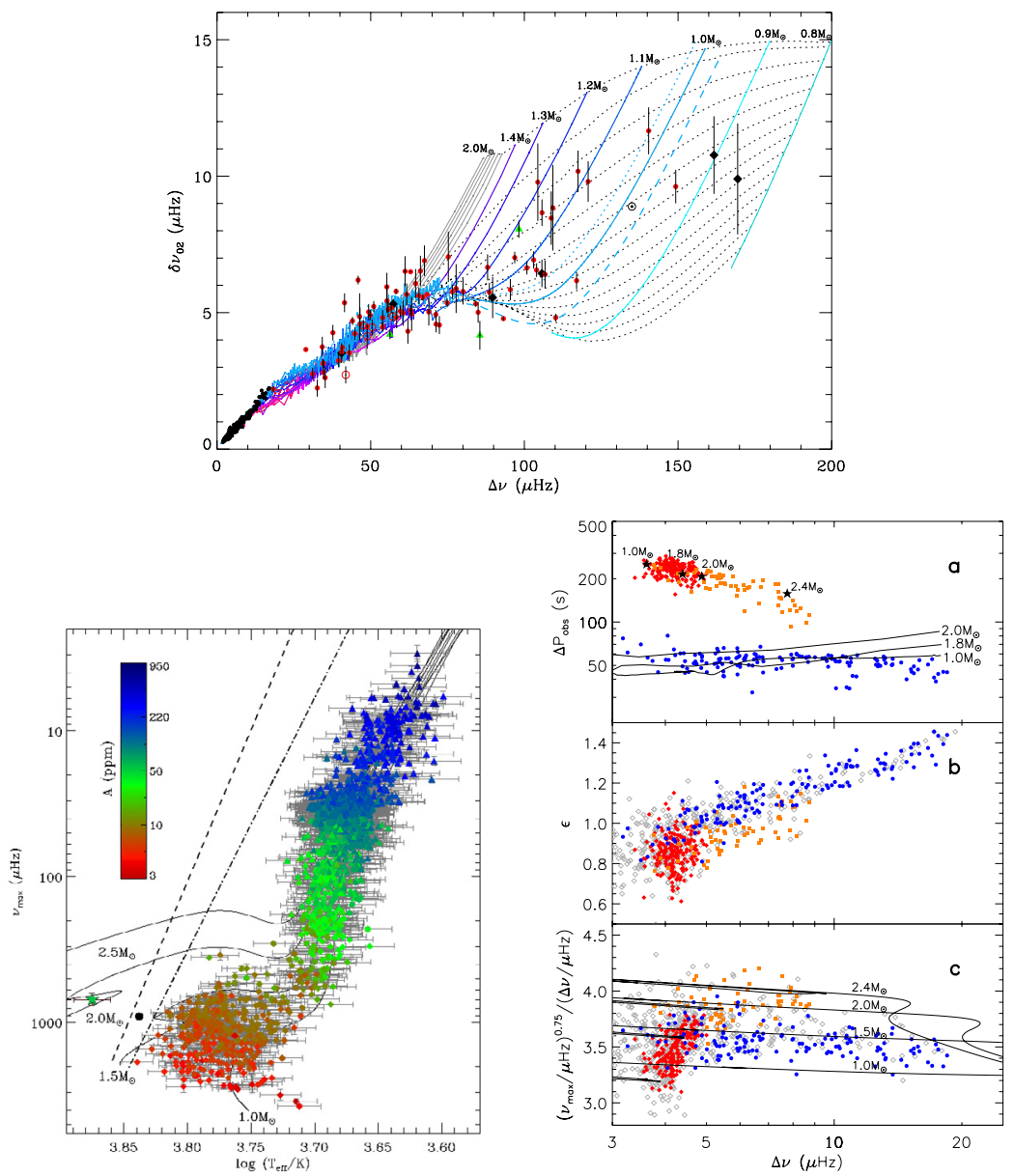

Fig. 2. a) KEPLER stellar seismic diagram using $\ell=0$ and 2. From White et al. (2011). b) Position of the peak maximum of the power spectrum versus temperature of more than 1000 red giants, the amplitude variability shows different populations (Huber et al. 2011). c) Period of the gravity modes of red giants showing clearly in red the helium burning and the hydrogen burning in blue. From Bedding et al. (2011).

mode observations of about 1000 red giants (Figs. 2b and 2c) bring to light the hydrogen and helium burning phases, well separated by their gravity periods or by the maximum of their power spectrum (Huber et al. 2011; Bedding et al. 2011). So a new potential of asteroseismology is definitively shown.

\section{Open problems deduced from seismic observations}

These seismic missions will reveal the internal dynamics of stars, but a good identification of frequencies is based on a proper microscopic physics. Unfortunately 
some uncertainties on energy transfer (linked to plasma opacities) have been identified and two cases have retained our attention (Turck-Chièze et al. 2009):

1) The extraction of the solar internal sound speed and rotation has revealed clear discrepancy with the classical solar theoretical predictions ( $\mathrm{K}$ for kernels of sound speed, density and rotation, see Christensen-Dalsgaard 2003):

$$
\begin{gathered}
\frac{\delta \nu_{n, \ell}}{\nu_{n, \ell}}=\int_{0}^{R}\left[K_{c}^{(n, \ell)}(r) \frac{\delta c}{c}(r)+K_{\rho}^{(n, \ell)}(r) \frac{\delta \rho}{\rho}(r)\right] d r+Q_{n, \ell}^{-1} G\left(\omega_{n, \ell}\right) \\
\delta \nu_{n, \ell, m}=m \int_{0}^{R} \int_{0}^{\pi} K_{\Omega}^{n, \ell, m}(r, \theta) \Omega(r, \theta) r d r d \theta .
\end{gathered}
$$

These discrepancies are not explained by the introduction of transport of momentum by rotation (Turck-Chièze et al. 2010; Turck-Chièze et al. 2011, see Turck-Chièze \& Couvidat 2011 for an overview). So the energy transfer stays clearly under suspicion. In parallel, the recent OPAS (Blancard et al. 2011) have revealed large differences in individual element spectra with previous calculations, exhibing some compensation between low Z nuclei and the iron group contributions that encourage experimental verifications.

2) The second case concerns massive stars. SPB and $\beta$ Cephei stars are interesting pulsators that might put important constraints on rotation and magnetic fields, two key actors for massive stars. Presently, the 40 observed stars in Kepler show acoustic and gravity modes probably up to $\ell=20$ (deformed stars) that are difficult to identify (Balona et al. 2011). The first difficulty comes from the iron group opacities, which drive their pulsations. The observed modes do not agree with the stability of modes deduced from the OP or OPAL tables, this fact suggests that those opacities are underestimated (Daszynska-Daszkiewicz \& Walczak 2010). The first analysis of the OPAC consortium confirms an underestimate of these opacities through comparison with more recent opacity calculations (Turck-Chièze et al. 2011; Gilles et al. 2011, 2012).

\section{Transfer of energy in stars}

It is why we have created an international consortium on opacities called OPAC to solve the microscopic problems mentioned above. This consortium is composed of astrophysicists and plasma physicists from different laboratories ${ }^{2}$. Our consortium 1) compares the existing tables OPAL and OP with 3 new calculations: OPAS (Blancard et al. 2011), SCO-RCG (Porcherot et al. 2011) and HULLAC (Bar-Shalom et al. 2001) for cases mentioned previously; 2) performs specific laser experiments to validate calculations: we have already realized several experiments

\footnotetext{
${ }^{2}$ S. Turck-Chièze (PI), C. Blancard, T. Caillaud, P. Cosse, T. Blenski, J.E. Ducret, J. Farriaut, G. Faussurier, D. Gilles, F. Gilleron, G. Loisel, L. Piau, J.C. Pain, M. Poirier, C. Reverdin, V. Silvert, F. Thais, B. Villette from CEA in France, F. Delahaye, C. Zeippen from Obs. Meudon, France, S. Bastiani from Ecole Polytechnique, M. Busquet from ARTEP, USA, J. Guzik, D.P. Kilcrease, N.H. Magee from Los Alamos USA and J.W. Harris from AWE England.
} 
at LULI for iron, nickel, copper... in X and XUV domains (Loisel et al. 2009, 2011, see below), at relatively low temperature, around $20-30 \mathrm{eV}(1 \mathrm{eV}=11600 \mathrm{~K})$. We prepare new experiments on PETAL+LMJ (maybe ORION) for solar-like radiative stellar conditions to complete $\mathrm{Z}$ pinch experiments (Bailey et al. 2007); 3) will also improve the stellar calculations and will study their impact on stellar evolution. Finally we will deliver new validated tables to the stellar community.

\subsection{First lessons from opacity calculation comparisons}

OP mean Rosseland values are generally greater than OPAL ones in the temperature range corresponding to the iron opacity bump (Fig. 2 of Turck-Chièze et al. 2011). In OP tables the chromium contribution of the iron group reaches $16 \%$, nickel $25-30 \%$, iron $65 \%$ of the total opacity. In fact, the main difficulty of the opacity tables is due to very complex calculations that justify approximations and even simplification to build tables in a reasonable time. Our comparison of OP values to new calculations reveals that all the elements have not been calculated with the same quality level. The nickel OP contribution is certainly underestimated (lower than all the other recent code calculations), see Figure 1 of Gilles et al. (2012) that summarizes the first comparison. The iron case is probably also slightly underestimated. The origins of the discrepancies have been looked for using HULLAC detailed computations (see Gilles et al. these proceedings): some transitions that play a role in the mean Rosseland values have not been taken into account in OP tables, moreover the interaction of configuration plays a non negligible role and seems to be absent from OPAL calculations, see the comparison with the Da Silva experiment by Whittaker \& Tallents (2009) and Figure 2 of Gilles et al. (2012). So in order to get more accuracy on the iron peak, one needs to improve the whole calculations of the different elements that participate to it. It is not only sufficient to consider all the necessary transitions, one needs also to have a proper treatment of the broadening of the lines and of the plasma interaction. This is why experiments are important to validate the theoretical results.

\subsection{First lessons from experimental results}

Several experiments have been done in the past on iron, used to check the calculations (see previous subsection). We have just performed at LULI2000 complementary experiments around $30 \mathrm{eV}$, measuring XUV spectrum up to $200 \mathrm{eV}$ for neighbored nuclei ( $\mathrm{Cr}, \mathrm{Fe}, \mathrm{Ni}$ and $\mathrm{Cu}$ ) with the objective to put complementary constraints on the calculations. We use two complementary lasers: a ns laser delivers energy between $300-500 \mathrm{~J}$ in a $500 \mathrm{ps}$ duration pulse. This laser is used to irradiate a gold cavity (hohlraum) on which a foil of the studied element (iron, nickel...) is deposited and heated. After a delay chosen to get the required density and temperature, a picosecond laser interacts with a backlighter foil to produce $\mathrm{X}$-rays in a short pulse $(10-30 \mathrm{ps})$ to probe the formed plasma. The transmission spectrum of the photons: $T(\nu)=\exp ^{(-\kappa(\nu) \rho r)}$ where $\mathrm{r}$ is the thickness of the foil, is measured by a streak camera placed behind a specifically designed XUV-ray spectrometer. A detailed description of the experimental set up and of the experiment 

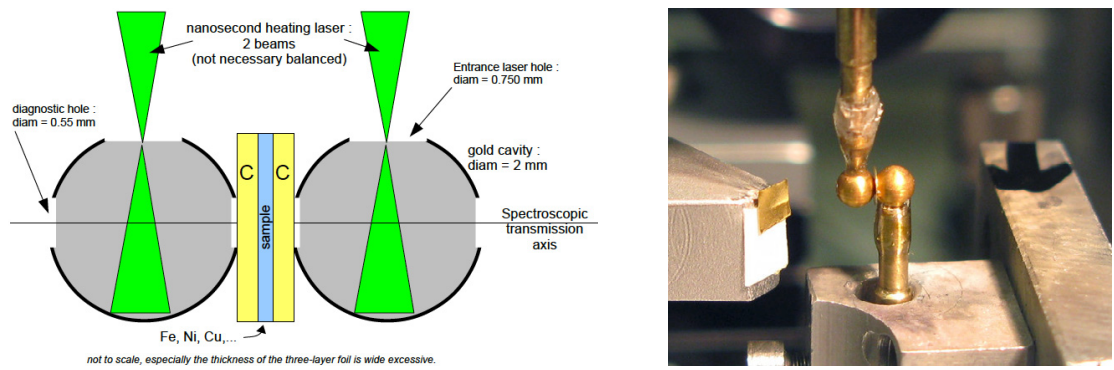

Fig. 3. Principle of the double cavity realized for the XUV experiment at LULI2000 with two gold cavities to limit the temperature and density gradients in the foil.
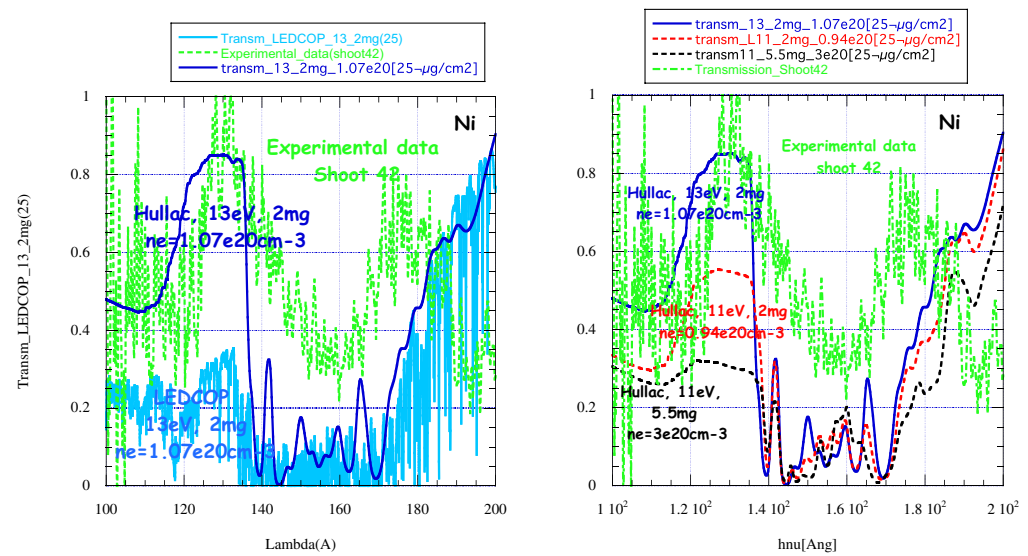

Fig. 4. Nickel transmission spectra at $13 \mathrm{eV}$ for LEDCOP, HULLAC (continuous lines) and preliminary analysis of experiments done on LULI 2000 (dotted lines). The sensitivity to temperature and density is shown on the right panel for HULLAC calculations.

analysis can be found in Loisel (2011). The quality of these measurements requires to form a plasma in LTE at the required conditions (equivalent ionization conditions than stellar conditions). This relies upon a good simulation of the whole experiment, in order to probe the foil at the best moment to get the appropriate temperature without pollution due to the emission of the gold of the cavity. Moreover, the rapid expansion of the foil during the heating is limited by placing the foil between two thin samples of a low $\mathrm{Z}$ material (here carbon), playing the role of inertial confinement to prevent an uncontrolled hydrodynamic expansion resulting in a dramatically decrease of the density. We measure the radiative temperature of the $\mathrm{X}$ radiation in the cavity thanks to a 12 channels spectrometer which measures X-rays energy (Bourgade et al. 2001). We limit the temperature gradient below $10 \%$ inside the foil in separating the incident ns beam in two parts, and inserting the foil between two cavities (Fig. 3). The streak camera gives a 
time dependence of the phenomenon (resolution of about $50 \mathrm{ps}$ ), in order to discriminate between backlight signal and self-emission of the cavity. Figure 4 shows preliminary comparisons with new calculations and the high sensitivity of the calculations to the temperature of the foil. The quality of the experiments relies on well calibrated spectrometer and detector.

\section{References}

Baglin, A., et al., 2006, AIP Conf. Proc., 895, 201

Bailey, J., et al., 2009, Phys. Plasma, 16, 058101

Balona, L.A., et al., 2011, MNRAS, 413, 2651

Bar-Shalom, A., Klapisch, M., \& Oreg, J., 2001, JQSRT, 71, 169

Blancard, C., Cosse, P., \& Faussurier, G., 2011, ApJ, 745, 10

Bedding, T., et al., 2001, ApJ, 549, L105

Bedding, T., et al., 2010, ApJ, 713, 935

Bedding, T., et al., 2011, Nature, 471, 6088

Bourgade, J.L., et al., 2001, Rev. Sci. Inst., 72, 1173

Chaplin, W.J., et al., 2010, ApJ, 713, 169

Christensen-Dalsgaard, J., 2003, Lecture notes on stellar oscillations, fifth edition

Daszynska-Daszkiewicz, \& Walczak, 2010, MNRAS, 403496

Gabriel, A.H., et al., 1995, Sol. Phys., 162, 61

Gilles, D., Turck-Chièze, S., et al., 2011, HEDP, 7, 312 and poster in these proceedings

Gilles, D., Turck-Chièze, S., et al., 2012, IFSA proceedings, in press

Gilliland, R.L., et al., 2010, ApJ, 713, L160

Huber, et al., 2011, ApJ, 743, 143

Kjeldsen, et al., 2008, AIP Conf. Proc., 1043, 365

Loisel, G., et al., 2009, HEDP, 5, 173

Loisel, G., 2011, Thesis Paris XI University, Orsay

Martic, M., et al., 2004, A\&A, 418, 295

Porcherot, Q., et al., 2011, HEDP, 7, 234

Scherrer, P.H., et al., 1995, Sol. Phys., 162, 129

Scherrer, P.H., et al., 2012, Sol. Phys., 275, 207

Stello, D., Kjeldsen, H., \& Bedding, T.R., 2007, ASP Conf. Ser., 366, 247

Turck-Chièze, S., Garcia, R.A., Couvidat, S., et al., 2004, ApJ, 604, 455

Turck-Chièze, S., et al., 2009, HEDP, 5, 132

Turck-Chièze, S., Palacios, A., Marques, J., \& Nghiem, P.A.P., 2010, ApJ, 715, 153

Turck-Chièze, S., \& Couvidat, S., 2011, Report Progr. Phys., 74, 086901

Turck-Chièze, S., Piau, L., \& Couvidat, S., 2011, ApJ, 731, L29

Turck-Chièze, S., et al., 2011, Ap\&SS, 336, 103

White, T.R., et al., 2011, ApJ, 742, L3

Whittaker, D.S., \& Tallents, G.J., 2009, MNRAS, 400, 1808 